\title{
WARS2 Gene
}

National Cancer Institute

\section{Source}

National Cancer Institute. WARS2 Gene. NCI Thesaurus. Code C132152.

This gene is involved in tRNA maturation. 